\title{
Improving ambiguity resolution by applying ionosphere corrections from a permanent GPS array
}

\author{
Dennis Odijk \\ Department of Mathematical Geodesy and Positioning, Delft University of Technology, Thijsseweg 11, 2629 JA Delft, The Netherlands
}

(Received January 6, 2000; Revised June 26, 2000; Accepted July 5, 2000)

Fast and high precision relative GPS positioning over distances up to $100 \mathrm{~km}$ is mainly limited by errors in the GPS signals due to propagation through the Earth's ionosphere. With permanent GPS arrays, which are present in many countries nowadays, it becomes possible to correct a user's GPS measurements to a certain extent for these ionospheric delays. A way to do so is to interpolate the ionospheric delays which have been estimated from the network of permanent stations. When these 'interpolated corrections' are applied to the user's data, the ionospheric delays may be reduced, which may lead to an improved ambiguity resolution for his (long) baseline.

\section{Introduction}

Integer GPS carrier phase ambiguity resolution is a prerequisite to get very precise positioning results using short observation time spans. Ionospheric errors in the relative GPS observations over baselines of medium lengths (longer than $10 \mathrm{~km}$ ) hamper a fast estimation of the integer carrier phase ambiguities. This problem is expected to grow in the coming maximum of the sunspot cycle (expected in the years 2000-2002). If a permanent GPS array is within the vicinity, then this can be an outcome, as it is possible to estimate precise ionospheric delays from the network. These estimates can then be interpolated for an arbitrary location within the surroundings of the network and these interpolated values can be provided to users to correct their GPS measurements. In the Netherlands such an ionosphere interpolation technique will be part of the so-called Virtual GPS Reference Station concept (as explained in van der Marel, 1998). This means that the observation data of the Dutch permanent stations are transformed to a location, which is approximately the position of the user's antenna, and these data are corrected for the errors that may be expected at the user's location. Next, the user processes these virtual data together with the data of his receiver as an ordinary 'short baseline'.

The purpose of this article is to gain insight in how well the ionospheric errors that are interpolated from the permanent stations coincide with the true ionospheric errors at the location of the virtual station. To test this performance, provisional tests have been carried out with samples of data of the Southern California Integrated GPS Network (SCIGN). The reason of not using data of the permanent network in the Netherlands, is that the SCIGN network is very dense and no additional measurements were necessary. Besides, the SCIGN data are very well archived and freely available at http://www.scign.org. Furthermore, the geomag-

Copy right (C) The Society of Geomagnetism and Earth, Planetary and Space Sciences (SGEPSS); The Seismological Society of Japan; The Volcanological Society of Japan; The Geodetic Society of Japan; The Japanese Society for Planetary Sciences. netic latitude of the SCIGN network is close to that of the Dutch network (both are located in the geomagnetic midlatitudes) and due to the geomagnetic latitude dependence of the ionospheric activity similar ionospheric errors may be expected (see Klobuchar, 1991).

\section{Fast Positioning in a Permanent GPS Array}

The permanent GPS array in the Netherlands, which is called the AGRS.NL (Active GPS Reference System), consists of five stations, with separations of 100-200 km. As a consequence, for a user operating in the Netherlands, the distance to the nearest AGRS.NL reference station can be as large as $100 \mathrm{~km}$. This means that in order to obtain a precise position, most of the times he has to account for significant ionospheric delay errors in his measurements.

To explain this, consider the general mathematical model to process (relative) GPS observations. The model of dualfrequency phase and code observation equations in doubledifference (DD) mode reads for at least two receivers simultaneously tracking at least four satellites at observation epoch $i$ :

$$
\begin{aligned}
& \phi_{1}(i)=B(i) b+\lambda_{1} a_{1}-I(i) \\
& \phi_{2}(i)=B(i) b+\lambda_{2} a_{2}-\left(\lambda_{2}^{2} / \lambda_{1}^{2}\right) I(i) \\
& p_{1}(i)=B(i) b+I(i) \\
& p_{2}(i)=B(i) b+\left(\lambda_{2}^{2} / \lambda_{1}^{2}\right) I(i)
\end{aligned}
$$

In this model $\phi_{1}$ and $\phi_{2}$ are the 'observed-minuscomputed' DD phase observables (in units of meters rather than cycles) on $\mathrm{L} 1$ and $\mathrm{L} 2$ respectively and $p_{1}$ and $p_{2}$ denote the observed-minus-computed DD code (pseudo-range) observables on L1 and L2. The vector $b$ represents the increments of the components of the baseline coordinates, whereas the matrix $B(i)$ contains the receiver-satellite unit vectors. The known wavelengths are denoted as $\lambda_{1}$ and $\lambda_{2}$, and $a_{1}$ and $a_{2}$ are the unknown but time-invariant DD phase ambiguities. Furthermore, $I(i)$ is the DD form of the unknown 
slant ionospheric delays on the L1-frequency. Note that in model (1) no unknowns for tropospheric, orbit, multipath as well as other errors have been introduced. These are supposed to be sufficiently small or accounted for by models or corrections.

Resolution of the integer DD ambiguities makes precise positioning feasible using very short observation time spans. Therefore, to model (1) integer constraints are added, which are denoted as $a_{j} \in Z,(j=1,2)$, with $Z$ being the space of integer numbers. However, the inclusion of the ionospheric unknowns in model (1) hampers a quick resolving of the integer ambiguities. Only in case of moderate ionospheric circumstances at the mid-latitude regions, for baselines shorter than about $10 \mathrm{~km}$ the relative ionospheric delays are so small that they may be neglected. For such short baselines it has already been extensively shown in the literature that the (correct) integer ambiguities can be estimated in very short time spans, see e.g. Tiberius et al. (1997). For precise positioning purposes within the AGRS.NL network however, usually a user has to account for the non-negligible ionospheric delays in his observations. If the ionospheric delays are estimated from the observations simultaneously with the other parameters according to model (1), the minimum time to successfully resolve the integer ambiguities is typically 30 minutes or more. For many applications this time span will be too long. A better idea is to reduce the ionospheric errors in the observations and not to estimate any parameters, which can be realized by correcting the user's data with interpolations of the ionospheric delays estimated at the permanent stations.

\section{Interpolation of Network Ionospheric Delays}

In this section it will be explained how the ionospheric interpolations at the site of a user can be generated from the GPS data at the permanent stations.

In a first step, the observed data at the permanent stations are processed according to the model (1). Using a relatively long time span of data (i.e. about 1 hour with a sampling of $30 \mathrm{sec}$.) and the known positions of the permanent stations, the integer ambiguities can be resolved correctly. In a next step, with the integer ambiguities held fixed, estimates for the ionospheric delays between these permanent stations are obtained. These so-called ambiguity-fixed estimates of the DD ionospheric delays (which have a very high precision if the correct ambiguities have been resolved) form the input of an interpolation algorithm, which is based on the spatial correlation between these relative ionospheric delays.

This interpolation for a certain user location $x$ with respect to a pivot location (here: permanent station 1) is carried out for each individual observation epoch and each satellite $s$ (with respect to a pivot satellite $p$ ). The interpolated DD ionospheric delay, $\bar{I}_{1 x}^{p s}$, can be computed, according to (van der Marel, 1998), as:

$$
\begin{aligned}
\bar{I}_{1 x}^{p s}= & \left(\begin{array}{cccc}
c_{x 1}^{s} & c_{x 2}^{s} & \cdots & c_{x n}^{s}
\end{array}\right) . \\
& \left(\begin{array}{cccc}
c_{11}^{s} & c_{12}^{s} & \cdots & c_{1 n}^{s} \\
c_{21}^{s} & c_{22}^{s} & \cdots & c_{2 n}^{s} \\
\vdots & \vdots & \ddots & \vdots \\
c_{n 1}^{s} & c_{n 2}^{s} & \cdots & c_{n n}^{s}
\end{array}\right)^{-1}\left(\begin{array}{c}
\hat{I}_{11}^{p s} \\
\hat{I}_{12}^{p s} \\
\vdots \\
\hat{I}_{1 n}^{p s}
\end{array}\right)
\end{aligned}
$$

where $\hat{I}_{1 r}^{p s}(r=1, \ldots, n)$ are the least-squares estimates of the DD ionospheric delays at the $n$ permanent stations and $c_{k t}^{s}$ is a spatial covariance function. Note that in the interpolation algorithm (2) the DD ionospheric delay at the pivot station $\hat{I}_{11}^{p s}$ has been added while this, by definition, equals zero. The reason for doing this is to take the geometry at the pivot station into account. Furthermore, note when applying the algorithm (2) for the locations of the permanent stations, their ionospheric input values are obtained: $\bar{I}_{1 r}^{p s}=\hat{I}_{1 r}^{p s}(r=$ $1, \ldots, n)$.

Important to the performance of the interpolation is the choice of the spatial covariance function $c_{k t}^{s}$. In the tests which will be described in Section 5 a spatial covariance function is assumed, which is a linear function of the distances between the ionospheric pierce points of the permanent stations: $c_{k t}^{s}=l_{\max }-l_{k t}^{s}$, where $l_{k t}^{s}$ is the geometric distance between the ionospheric points of receivers $k$ and $t$ and with $l_{\max }=\infty$. Note that a ionospheric pierce point is the intersection of the receiver-satellite line of sight with a single ionosphere layer (see Georgiadou and Kleusberg, 1988), which is here assumed sphere-like at a height of 350 $\mathrm{km}$ above the Earth, see Fig. 1. It is known that at a height of $350 \mathrm{~km}$ the GPS signals are mostly affected by the free electrons in the ionosphere and therefore the interpolation is performed at this 'ionosphere level' and not at ground level. The spatial covariance is chosen such that the covariance between two points is decreasing when the distance between these points is increased. This assumption is supposed to be valid as the absolute ionospheric delays for receivers at relatively close distance (maximum $200 \mathrm{~km}$ ) tend to be very similar. The setting of $l_{\max }=\infty$ in the spatial covariance function has been done to make the interpolation results independent of the choice of pivot station. Although an infinite value is not allowed considering the single covariance function, it is allowed when it is used in the interpolation algorithm (2).

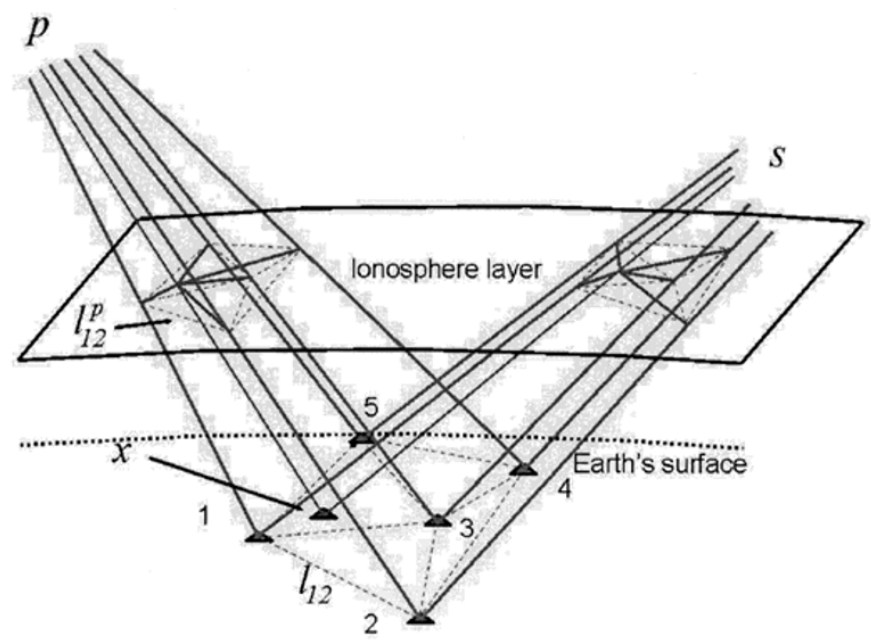

Fig. 1. Interpolation on a single ionosphere layer of the ionospheric delays estimated from the network of 5 permanent stations to a user station $x$. 


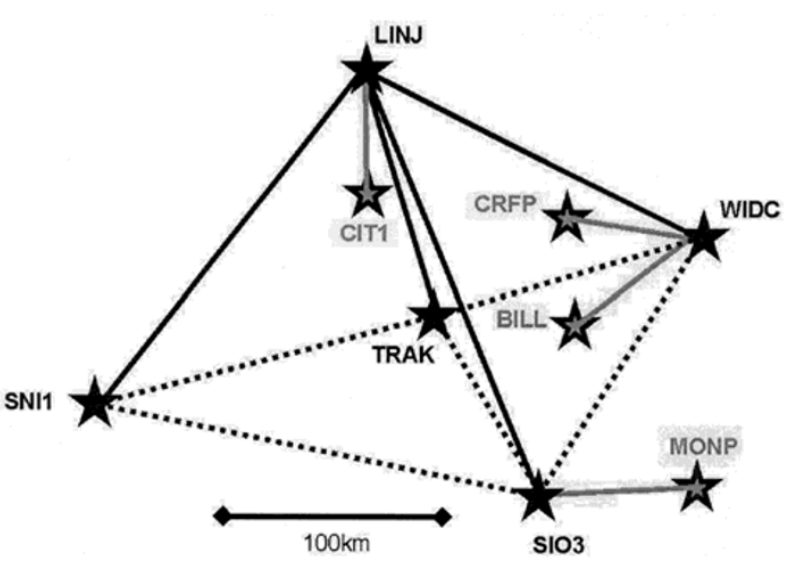

Fig. 2. Used stations of the SCIGN network in the tests: LINJ-TRAK-WIDC-SIO3-SNI1 (black stars-the 'permanent' stations) and CIT1-CRFP-BILL-MONP (grey stars-the 'user' stations). Note that the dotted lines are dependent baselines and that the user baselines all are all formed with the closest permanent station as reference station.

\section{Ambiguity Resolution with the Ionospheric Corrections Applied}

The topic of this article is to study how well the interpolation is able to predict the 'real' DD ionospheric delays at a position within a permanent network, even in periods of increasing ionospheric activity. This performance will be measured in terms of shortening of the time to successfully fix the integer ambiguities when the interpolated corrections are applied.

For this purpose, data from the SCIGN network has been used. In Fig. 2 the configuration of the stations used in the tests is shown. The SCIGN stations which are assigned as 'permanent' stations in the tests are 100-200 km separated from each other and the assigned 'user' stations all lie within 50-80 km from the nearest 'permanent' station. Note that station MONP lies outside of the network.

In a first step of the tests, ionospheric delays between the 'permanent' stations (pivot station LINJ) have been estimated for 1 hour of data collected at $30 \mathrm{sec}$. sampling interval according to model (1), with some modifications: no estimation of baseline coordinates (they are accurately known in case of a permanent network), and extension of the model with a tropospheric zenith delay parameter per station, because of considerable height differences between the permanent stations.

Next, the interpolated corrections at the user stations were computed using (2) and applied to the data of the user stations and their closest permanent stations. To measure the improvement of ambiguity resolution, the following procedure has been used:

1. Estimate a reference set of integer ambiguities.

A reference set of integer ambiguities ("ground-truth") at each user baseline was obtained using model (1), thus with ionospheric parameters estimated. These ambiguities could be resolved as the full hour of observations was processed. (Of course, in real practice these reference ambiguities cannot be obtained as only data of short time spans are available, and one has to rely on other ambiguity validation techniques.)

2. Process short time spans with and without interpolated corrections and compute ambiguity success rates.

Next, the 1-hour data sets at the user stations were divided into smaller windows to test whether the same integers as in step 1 could be resolved without the estimation of ionospheric parameters, but with the data a priori corrected. The selected time windows are: 1 epoch (instantaneous ambiguity resolution), 12 epochs (time span $5 \mathrm{~min}$.) and 20 epochs (10 min.). The estimated integers in this step were compared with those of step 1 and a success rate was obtained by taking the ratio of the number of correct ambiguity sets and the total number of ambiguity sets. To gain insight in the improvement for each baseline also integer success rates were estimated when the data were processed without estimation of ionospheric paremeters and without any corrections applied.

In all tested cases, the assumed stochastic model of the observations is rather simple: all observations were uncorrelated assumed with a standard deviation of $3 \mathrm{~mm}$ for the (undifferenced) phase and $30 \mathrm{~cm}$ for the (undifferenced) code observations. For all processing the GPSveQ software of the Delft University of Technology (de Jonge, 1998) was used, which contains the $L A M B D A$-method for least-squares estimation of the integer ambiguities (see Teunissen, 1993).

\section{Test Results}

The results of two tests will be described. The first data set was collected in winter on 1 January 1999, during the time span 20:00:30-21:00:00 UTC (12:00:30-13:00:00 local time). This data set was selected because during this period ionospheric activity was disturbed due to the occurrence of 'travelling ionospheric disturbances' (TIDs). These TIDs mainly occur during winter months around local noon and cause a sudden change in the size of the relative ionospheric delays (see Wanninger, 1999). The second data set was collected in summer on 24 June 1999 and it is known that during this period the ionospheric activity was much less disturbed. Essentially, the same stations were used in both tests, the only difference being in the use of user station BILL rather than CRFP (see Fig. 2).

\subsection{Test data set 1: 1 January 1999}

In Fig. 3 time-series of the estimated DD ionospheric delays are given for the permanent stations. In this test 6 satellites have been used and the double-differences all hold with respect to PRN2. From the graphs one can see that the timeseries are smooth but fluctuating which is probably caused by TIDs.

Next, in Fig. 4 for the three user baselines the time-series of the estimated DD ionospheric delays with and without the application of the interpolated corrections are shown. In Table 1 for each of these baselines the ambiguity success rates are given. From the table we may conclude that without the corrections applied for most of the times the ionospheric errors are way too large to successfully fix the integer ambiguities. Even when the window lengths are increased, the ionospheric errors do not tend to 'average out' in most of the ambiguity solutions. Notice that for the baseline which is 'outside' the network (SIO3-MONP) in none of the windows the correct integers could be resolved. This poor result is not surprising considering the length of the tested baselines. Because the relative ionospheric delays are known to increase with baseline length, the 'best' results were obtained 

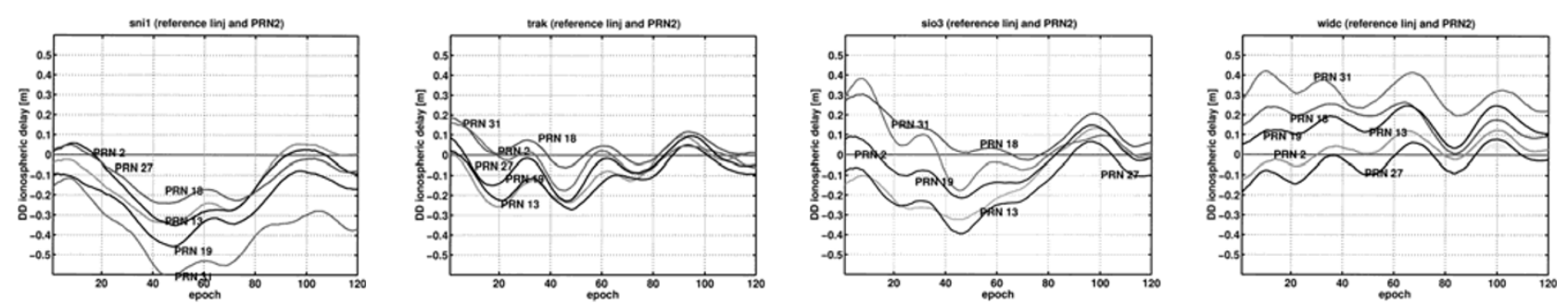

Fig. 3. Estimated DD ionospheric delays at permanent stations (from left to right: SNI1-TRAK-SIO3-WIDC) with respect to pivot station LINJ and pivot satellite PRN 2 on 1 January 1999, 20:00:30-21:00:00 UTC.

Table 1. Ambiguity resolution success rates with and without ionospheric corrections applied (1 January 1999).

\begin{tabular}{lccccccc}
\hline \multirow{2}{*}{$\begin{array}{c}\text { Ambiguity } \\
\text { Success-rate }\end{array}$} & \multicolumn{3}{c}{ Without interpolated corrections } & & \multicolumn{3}{c}{ With interpolated corrections } \\
\cline { 2 - 3 } \cline { 7 - 9 } & $\begin{array}{c}\text { LINJ-CIT1 } \\
(58 \mathrm{~km})\end{array}$ & $\begin{array}{c}\text { WIDC-CRFP } \\
(66 \mathrm{~km})\end{array}$ & $\begin{array}{c}\text { SIO3-MONP } \\
(78 \mathrm{~km})\end{array}$ & & $\begin{array}{c}\text { LINJ-CIT1 } \\
(58 \mathrm{~km})\end{array}$ & $\begin{array}{c}\text { WIDC-CRFP } \\
(66 \mathrm{~km})\end{array}$ & $\begin{array}{c}\text { SIO3-MONP } \\
(78 \mathrm{~km})\end{array}$ \\
\hline Instantaneous & $7 \%$ & $0 \%$ & $0 \%$ & & $31 \%$ & $44 \%$ & $9 \%$ \\
5 Minutes & $17 \%$ & $8 \%$ & $0 \%$ & & $67 \%$ & $67 \%$ & $17 \%$ \\
10 Minutes & $50 \%$ & $17 \%$ & $0 \%$ & & $100 \%$ & $83 \%$ & $17 \%$ \\
\hline
\end{tabular}
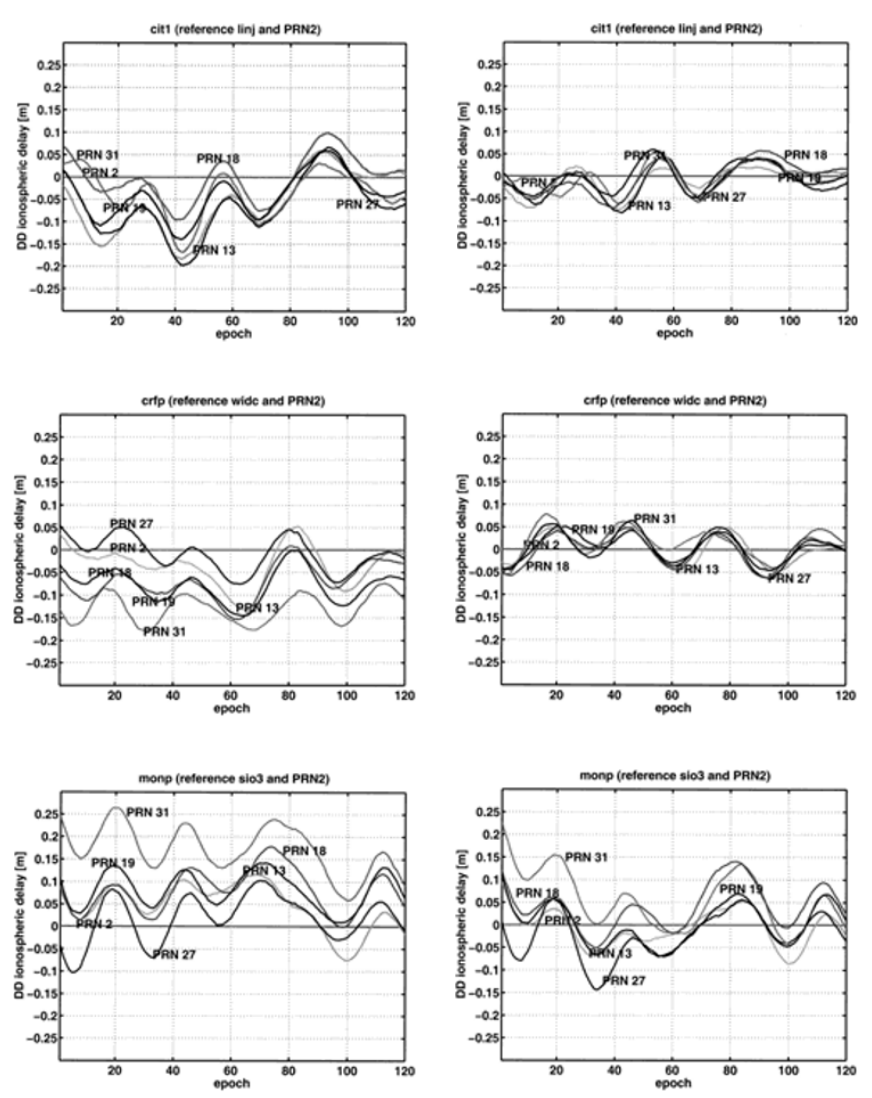

Fig. 4. DD ionospheric delay in data (graphs on the left) and residuals after correction from interpolation (graphs on the right) for baselines LINJ-CIT1 (58 km; top), WIDC-CRFP (66 km; middle) and SIO3-MONP (78 km; bottom). for the shortest baseline LINJ-CIT1.

After correcting the data with the interpolated corrections the success rates increased considerably (see Table 1), though not for all time windows the correct integers could be resolved, not even with $10 \mathrm{~min}$. of data (baseline WIDCCRFP). Furthermore, for the baseline outside the network (SIO3-MONP), the success rate improved only marginally: from $0 \%$ to $17 \%$ at maximum. This last poor result can be explained from the fact that the interpolation scheme does not perform well when 'extrapolating' the ionospheric delays of the permanent stations to locations outside the network.

\subsection{Test data set 2: 24 June 1999}

The ionosphere interpolation was also tested on the second data set, measured on 24 June 1999. See Fig. 5 for the time-series of the DD ionospheric delays at the 'permanent' stations. Notice that due to a less disturbed ionospheric activity than on 1 January 1999, these time-series are more constant. Furthermore, note that for this test only 4 satellites were used in the computations, as to test the performance with a minimum number of satellites.

Considering the graphs of Fig. 6 it is obvious that the magnitude of the DD ionospheric delay for the three baselines after correction is much smaller than for the data set of 1 January 1999. This can also be seen from the success rates when the corrections are applied. For the baselines LINJCIT1 and WIDC-BILL in more than $70 \%$ of the epochs it has become possible to estimate the correct integers instantaneously, against about $30 \%$ on 1 January 1999. If measuring for at least $10 \mathrm{~min}$., for both baselines inside the network ambiguity resolution is successful for all windows. For the baseline outside the network, the success rates are higher than in the previous data set, but still too low for practical applications.

Reason for the poorer results of the test of 1 January 1999 is that the interpolation technique is not capable to completely 
Table 2. Ambiguity resolution success rates with and without ionospheric corrections applied (24 June 1999).

\begin{tabular}{lcccccccc}
\hline \multirow{2}{*}{$\begin{array}{c}\text { Ambiguity } \\
\text { Success-rate }\end{array}$} & \multicolumn{3}{c}{ Without interpolated corrections } & & \multicolumn{3}{c}{ With interpolated corrections } \\
\cline { 2 - 3 } & $\begin{array}{c}\text { LINJ-CIT1 } \\
(58 \mathrm{~km})\end{array}$ & $\begin{array}{c}\text { WIDC-BILL } \\
(74 \mathrm{~km})\end{array}$ & $\begin{array}{c}\text { SIO3-MONP } \\
(78 \mathrm{~km})\end{array}$ & & $\begin{array}{c}\text { LINJ-CIT1 } \\
(58 \mathrm{~km})\end{array}$ & $\begin{array}{c}\text { WIDC-BILL } \\
(74 \mathrm{~km})\end{array}$ & $\begin{array}{c}\text { SIO3-MONP } \\
(78 \mathrm{~km})\end{array}$ \\
\hline Instantaneous & $23 \%$ & $7 \%$ & $0 \%$ & & $73 \%$ & $98 \%$ & $19 \%$ \\
5 Minutes & $67 \%$ & $17 \%$ & $0 \%$ & & $92 \%$ & $100 \%$ & $42 \%$ \\
10 Minutes & $67 \%$ & $17 \%$ & $0 \%$ & & $100 \%$ & $100 \%$ & $50 \%$ \\
\hline
\end{tabular}
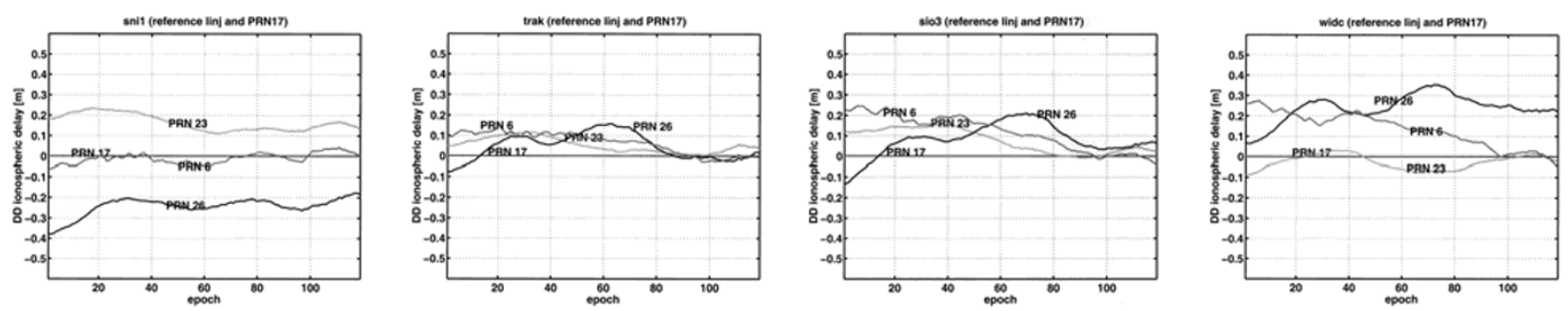

Fig. 5. Estimated DD ionospheric delays at permanent stations (from left to right: SNI1-TRAK-SIO3-WIDC) with respect to pivot station LINJ and pivot satellite PRN 2 on 24 June 1999, 20:00:30-21:00:00 UTC.
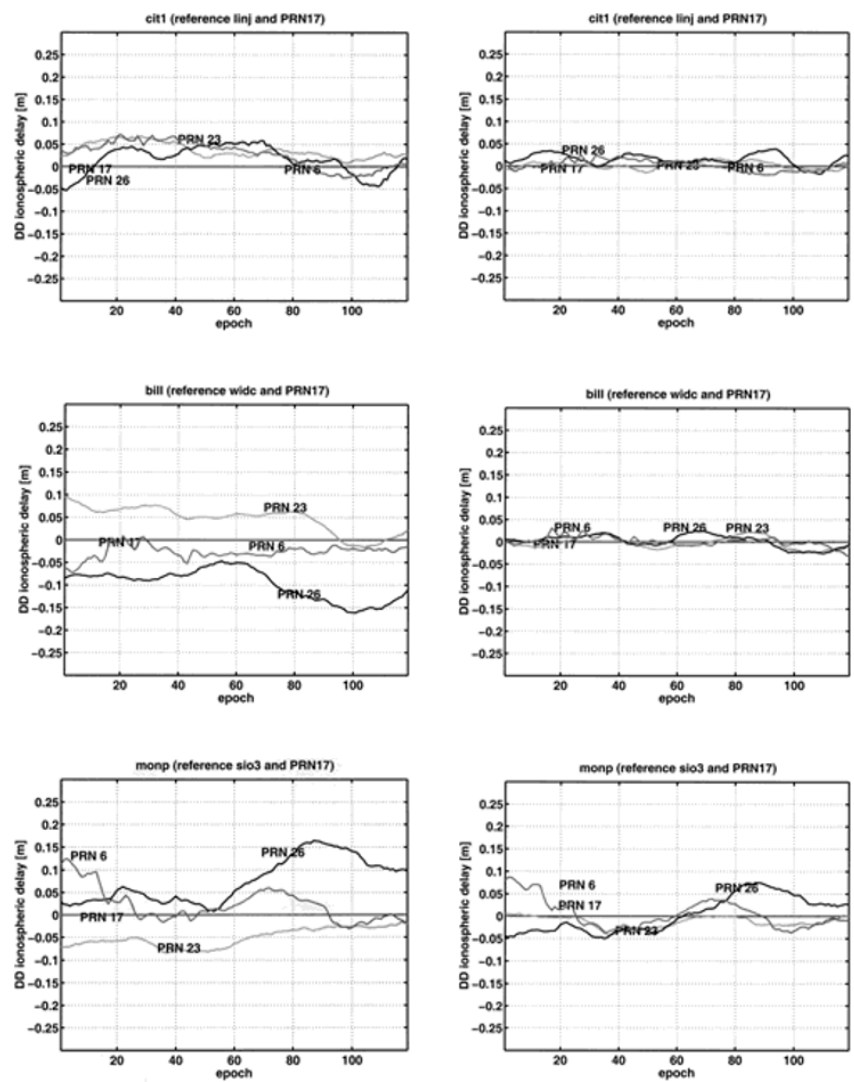

Fig. 6. DD ionospheric delay in data (graphs on the left) and residuals after correction from interpolation (graphs on the right) for baselines LINJ-CIT1 (58 km; top), WIDC-BILL (74 km; middle) and SIO3-MONP (78 km; bottom). reduce the effect of the TIDs in the data. When comparing the left-hand graphs and the right-hand graphs of Fig. 4, the fluctuating behaviour is still present in the residuals after correction.

\section{Concluding Remarks}

In this contribution provisional results have been shown of an interpolation technique in order to reduce the ionospheric delays in long GPS baseline processing. This interpolation will be part of the virtual reference station concept in the permanent GPS array in the Netherlands.

With respect to the performance of the ionospheric interpolation we may conclude the following: 1. Computations with data of the SCIGN network show that the interpolation technique indeed reduces the ionospheric errors in the user's baselines; 2 . For the considered baselines ambiguity resolution was significantly improved using $10 \mathrm{~min}$. of corrected data, but only if these baselines are located within the area of the permanent network and the ionospheric activity is not disturbed.

On basis of these conclusions, it is expected that for the near-future period of maximum solar and ionospheric activity the performance is not enough when one aims at fast and reliable ambiguity resolution over distances of $100 \mathrm{~km}$. Improvement of the performance may however be expected from 1. A densification of the permanent array; 2. An improved spatial covariance function; 3 . At the user's processing: a weighting of the ionosphere corrections (instead of a deterministic treatment).

Acknowledgments. This work was carried out under a contract with the Dutch Cadastre. Furthermore, the Southern California Integrated GPS Network and its sponsors, the W. M. Keck Foundation, NASA, NSF, USGS, SCEC, are acknowledged for providing the data used in this study. Finally, Frank Kleijer and Peter Joosten of the Delft University of Technology are thanked for assisting with the preparation of the manuscript. 


\section{References}

Georgiadou, Y. and A. Kleusberg, On the effect of ionospheric delay on geodetic relative GPS positioning, Manuscripta Geodaetica, 13, 1-8, 1988.

Jonge, P. J. de, A Processing Strategy for the Application of the GPS in Networks, 225 pp., Netherlands Geodetic Commission, Publications on Geodesy, 46, 1998.

Klobuchar, J. A., Ionospheric effects on GPS, GPS World, 4, 48-51, 1991.

Marel, H. van der, Virtual GPS reference stations in the Netherlands, Proc. ION GPS-98, 49-58, 1998.

Teunissen, P. J. G., Least-squares estimation of the integer GPS ambiguities,
Invited lecture, Sect. IV Theory and Methodology, IAG General Meeting, Beijing, August, 1993.

Tiberius, C. C. J. M., P. J. G. Teunissen, and P. J. de Jonge, Kinematic GPS: performance and quality control, Proc. KIS'97, 289-299, 1997.

Wanninger, L., The performance of virtual reference stations in Active Geodetic GPS-networks under solar maximum conditions, Proc. ION GPS-99, 1419-1427, 1999.

D. Odijk (e-mail: D.Odijk@geo.tudelft.nl) 